\title{
USING HARMONIC BALANCE METHOD FOR SOLVING FREQUENCY RESPONSE OF SYSTEMS WITH NONLINEAR ELASTIC FOUNDATION
}

\author{
Zaoral F.*, Ferfecki P.,"**
}

\begin{abstract}
The frequency response of the console, discretized by three-dimensional finite elements, excited by a pulsating surface distributed load and mounted on a bilateral nonlinear elastic foundation, reaction forces of which are described by the third-degree polynomial, was investigated. The solution was performed in a program library for solving the problems of steady-state vibration of nonlinear dynamic systems, which is being developed in the MATLAB software. To describe the frequency response curve, the Crisfield's continuous arc length method with the secant predictor was applied. The harmonic balance method using the Alternating Frequency-Time scheme was used to evaluate the non-linear forces in each iteration of the solution to solve the steady-state vibration response in every continuation increment.
\end{abstract}

\section{Keywords: Finite Element Method, Harmonic Balance, Arc Length, Nonlinear Elastic Foundation.}

\section{Introduction}

In practice, modeling of mechanical systems supported on an elastic foundation is a common problem in the construction industry when designing building foundations, in mining when designing mine reinforcements or in rail transport. A mathematical description of behavior of the elastic foundation usually causes that the computational model of a mechanical system, although otherwise linear, becomes nonlinear.

If there is a need to know the frequency response of such system, a necessity arises to use one of the continuation methods. Different variants of the arc length method are often used. Description of the frequency response then requires repeated solutions of the steady-state component of system displacements for varying excitation frequency. A possible way-out is the application of the harmonic balance method, which allows for the determination of steady-state response component directly, provided it has a periodic or quasi-periodic time course (Detroux et al., 2015).

Frequency response investigation is closely related to the problem of stability of the vibration response. However, investigation of the vibration response stability is not the focus of this work.

\section{Harmonic balance method}

The equation of motion of a dynamic system with a nonlinear elastic foundation, discretized by finite elements, is assumed to be in the form

$$
\mathbf{M} \ddot{\mathbf{x}}+\mathbf{B} \dot{\mathbf{x}}+\mathbf{K x}=\mathbf{p}_{s}+\mathbf{p}_{h}(t)-\mathbf{r}(\mathbf{x}, t),
$$

where $\mathbf{M}, \mathbf{B}$, and $\mathbf{K}$ are order n square matrices of mass, damping, and stiffness, respectively, $\mathbf{x}$, $\dot{\mathbf{x}}$, and $\ddot{\mathbf{x}}$ are vectors of displacement, velocity, and acceleration of individual mesh nodes, respectively, $\mathbf{r}(\mathbf{x}, t)$ is a vector of nonlinear forces via which the elastic foundation acts on the system, $\mathbf{p}_{s}$ is a vector of static forces and $\mathbf{p}_{h}(t)=\mathbf{p}_{h a} \cos (\omega t+\psi)$ is a vector of harmonic excitation forces, where $\mathbf{p}_{h a}$ is amplitude of force, $\omega$ is an angular excitation frequency, $t$ is the time and $\psi$ is a phase shift. Using the harmonic balance method,

\footnotetext{
* Ing. Filip Zaoral: IT4Innovations, VSB - Technical University of Ostrava, 17. listopadu 2172/15, 708 00, Ostrava-Poruba, CZ, filip.zaoral@vsb.cz

** Ing. Petr Ferfecki, PhD.: Department of Applied Mechanics, VSB - Technical University of Ostrava, 17. listopadu 2172/15, 70800 Ostrava-Poruba, CZ, petr.ferfecki@vsb.cz
} 
the steady-state component of the periodic response is approximated by the finite number $N_{F}$ of harmonic terms of the Fourier series in the form

$$
\mathbf{x}(t)=\frac{\mathbf{c}_{0}}{2}+\sum_{k=1}^{N_{F}}\left[\mathbf{c}_{k} \cos (k \omega t)+\mathbf{s}_{k} \sin (k \omega t)\right],
$$

where $\mathbf{c}_{0}, \mathbf{c}_{k}$, and $\mathbf{s}_{k}$ are vectors of the Fourier coefficients of absolute, cosine, and sine members, respectively. By substituting Eq. (2) and its time derivatives into Eq. (1) and comparing the corresponding absolute, sine, and cosine members, the equation of motion in the frequency domain can be obtained

$$
\mathbf{A}(\omega) \mathbf{u}=\mathbf{q}-\mathbf{b}(\mathbf{u}),
$$

here $\mathbf{A}(\omega)=\operatorname{diag}\left(\mathbf{K}, \mathbf{A}_{1}, \ldots, \mathbf{A}_{k}, \ldots, \mathbf{A}_{N_{F}}\right)$ is an order $n\left(2 N_{F}+1\right)$ square block diagonal matrix of dynamic stiffness. Vector $\mathbf{u}$ in Eq. (3) contains Fourier coefficients from Eq. (2) arranged as

$$
\mathbf{u}=\left[\begin{array}{lllllllll}
\mathbf{c}_{0} & \mathbf{c}_{1} & \mathbf{s}_{1} & \cdots & \mathbf{c}_{k} & \mathbf{s}_{k} & \cdots & \mathbf{c}_{N_{F}} & \mathbf{s}_{N_{F}}
\end{array}\right]^{\mathrm{T}},
$$

vector of linear forces q in Eq. (3) contains static and harmonic excitation forces and has the form

$$
\mathbf{q}=\left[\begin{array}{llllll}
\mathbf{p}_{s} & \sin (\psi) \mathbf{p}_{h a} & \cos (\psi) \mathbf{p}_{h a} & \mathbf{0} & \cdots & \mathbf{0}
\end{array}\right]^{\mathrm{T}},
$$

and to obtain a vector of nonlinear forces in the frequency domain $\mathbf{b}(\mathbf{u})$ in Eq. (3), Alternating FrequencyTime Scheme (AFT) with the linear operator of direct Fourier transformation $\mathbf{T}^{+}(\omega)$ can be used (Detroux et al., 2015),

$$
\mathbf{b}=\mathbf{T}^{+}(\omega) \mathbf{f},
$$

where the matrix $\mathbf{T}^{+}(\omega)$ can be obtained by calculating the Moore-Penrose pseudo-inverse of a matrix of the inverse Fourier transform $\mathbf{T}(\omega)$ with dimensions $n \times\left(2 N_{F}+1\right)$

$$
\mathbf{T}(\omega)=\left[\begin{array}{lllllllll}
0.5 \cdot 1 & \mathbf{t}_{c, 1} & \mathbf{t}_{s, 1} & \cdots & \mathbf{t}_{c, k} & \mathbf{t}_{s, k} & \cdots & \mathbf{t}_{c, N_{F}} & \mathbf{t}_{s, N_{F}}
\end{array}\right],
$$

here $\mathbf{1}$ is a vector of ones and $\mathbf{t}_{c, k}$ and $\mathbf{t}_{s, k}$ are function value vectors of cosine and sine functions, respectively, calculated at $N$ collocation points over a period of vibration.

Elements of the nonlinear force vector in the time domain $\mathbf{f}(\mathbf{x}, t)$ must be evaluated in each iteration of the solution of Eq. (3). To obtain the actual displacement vector $\mathbf{x}$, the following relation applies

$$
\mathbf{x}=\mathbf{T}(\omega) \mathbf{u} .
$$

\section{Arc length continuation method}

When using the arc length method to solve systems of equations in the deformation variant of the finite element method, the controlled parameter, which in dynamics is usually the angular excitation frequency, becomes unknown in addition to the system displacements and one more equation is added, fixing the incremental step length in the space of unknown variables.

The first step in finding a solution of the following increment in all variants of the arc length method is an initial estimate of the solution by a so-called predictor. The next step is an iterative refinement of the initial estimate of the solution by the so-called corrector. Eq. (9) can be defined between the vector of iterative displacements in the $j$-th iteration of the $i$-th increment $\delta \mathrm{u}_{j}^{i}$ and the iterative angular excitation frequency $\delta \omega_{j}^{i}$ (Batoz and Dhatt, 1979)

$$
\delta \mathbf{u}_{j}{ }^{i}=\mathbf{x}_{1}-\delta \omega_{j}^{i} \mathbf{x}_{2},
$$

where $\mathbf{x}_{1}$ and $\mathbf{x}_{2}$ are an orthogonal and parallel components of the displacement vector, respectively. To fix the length of the incremental step, Crisfield (1981) derived quadratic equation where one of its roots is an iterative angular excitation frequency $\delta \omega_{j}{ }^{i}$. Next, after calculating the iterative displacement vector $\delta \mathbf{u}_{j}{ }^{i}$ from Eq. (9) and updating the displacement vector and the angular excitation frequency

$$
\begin{aligned}
\mathbf{u}_{j+1}{ }^{i} & =\mathbf{u}_{j}^{i}+\delta \mathbf{u}_{j}^{i}, \\
\omega_{j+1}{ }^{i} & =\omega_{j}^{i}+\delta \omega_{j}^{i},
\end{aligned}
$$


all that remains is to decide on either the convergence of the solution or repeated iteration, according to an appropriate criterion. In most cases, the criterion of permissible error of unbalanced forces is satisfactory.

The different physical units of the displacement vector $\mathbf{u}$ and the angular excitation frequency $\omega$ can cause orders of magnitude differences between their numerical values, which in practice can lead to a significant increase in the number of iterations or even divergence of solution. For this reason, all physical quantities need to be scaled appropriately (Krack and Gross, 2019).

\section{Test problem}

The finite element mesh of the test problem with highlighted boundary conditions can be seen in Fig. 1 . It is a bracket pressed against a nonlinear elastic foundation by a constant pressure $p_{s}$ at surfaces colored blue. At the surfaces colored red, the bracket transmits a harmonically pulsating pressure load $p$ with a constant component $p_{m}$, which represents a bolt connection.
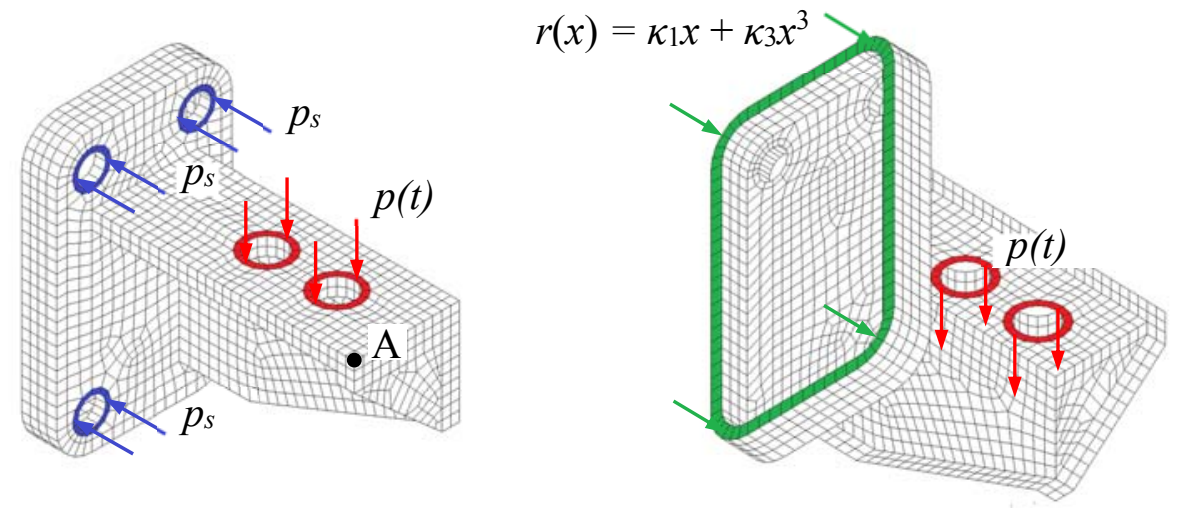

Fig. 1: Finite element mesh of the test problem with highlighted boundary conditions.

The foundation is modeled as bilateral in the form of a nonlinear force constraint represented by the surface load $r(x)$, which acts on the bracket perpendicular to the surface colored green and whose dependence on the displacement $x$ is described by the third-degree polynomial (Frydrýšek et al., 2014). The other degrees of freedom of nodes on this surface have zero displacements prescribed. The geometry of the bracket is discretized by 2,232 three-dimensional linear hexahedral finite elements with a total of 3,965 nodes. The material of the bracket is considered to be linearly elastic and isotropic. Damping is considered proportional. The physical parameters of the model are listed in Tab. 1.

Tab. 1: Physical parameters of the test problem.

\begin{tabular}{|l|c|c|c|}
\hline Parameter & Symbol & Value & Unit \\
\hline Young's modulus & $E$ & $6.9 \cdot 10^{10}$ & $\mathrm{~Pa}$ \\
\hline Poisson's number & $\mu$ & 0.33 & - \\
\hline Mass density & $\rho$ & $2.7 \cdot 10^{3}$ & $\mathrm{~kg} / \mathrm{m}^{3}$ \\
\hline Mass proportional damping coefficient & $\alpha$ & 100 & $1 / \mathrm{s}$ \\
\hline Stiffness proportional damping coefficient & $\beta$ & $5 \cdot 10^{-5}$ & $\mathrm{~s}$ \\
\hline Constant pressure & $p_{s}$ & $5 \cdot 10^{6}$ & $\mathrm{~Pa}$ \\
\hline Amplitude of distributed excitation pressure & $p_{a}$ & $1 \cdot 10^{6}$ & $\mathrm{~Pa}$ \\
\hline Mean value of distributed excitation pressure & $p_{m}$ & $5 \cdot 10^{6}$ & $\mathrm{~Pa}$ \\
\hline Linear stiffness coefficient of foundation & $\kappa_{1}$ & $1 \cdot 10^{9}$ & $\mathrm{~N} / \mathrm{m}^{3}$ \\
\hline Cubic stiffness coefficient of foundation & $\kappa_{3}$ & $5 \cdot 10^{15}$ & $\mathrm{~N} / \mathrm{m}^{5}$ \\
\hline
\end{tabular}

The harmonic balance method with successively 1, 2, 4, and 8 harmonic members $N_{F}$ was used to solve the steady-state component of forced vibration in each solution increment, always discretizing the vibration period to $N=3\left(2 N_{F}+1\right)$ collocation points. All physical parameters of the test problem were scaled due to numerical solution by multiplying all their length and time dimensions by a scaling parameter $s=1 \cdot 10^{3}$. 


\section{Results of the test problem and discussion}

The frequency response of the system was calculated at point A, see Fig. 1, at which the most significant response was expected. The effect of the elastic foundation on natural frequencies of the system is evident. The natural frequencies are now dependent on displacement and tend to increase in accordance with the stiffening characteristics of the foundation. In all cases, there can be several limit points identified on the frequency response curve, where the tangent to the curve is perpendicular to the horizontal axis. These points border areas of unstable response in which the vibration response of the system can be obtained neither by direct integration nor by measurement on the corresponding physical system.

For 4 harmonic terms of the Fourier series, no significant change in the shape of the frequency response curve was observed for the excitation frequency values from $300 \mathrm{~Hz}$ above, compared to the case with 8 harmonic terms.

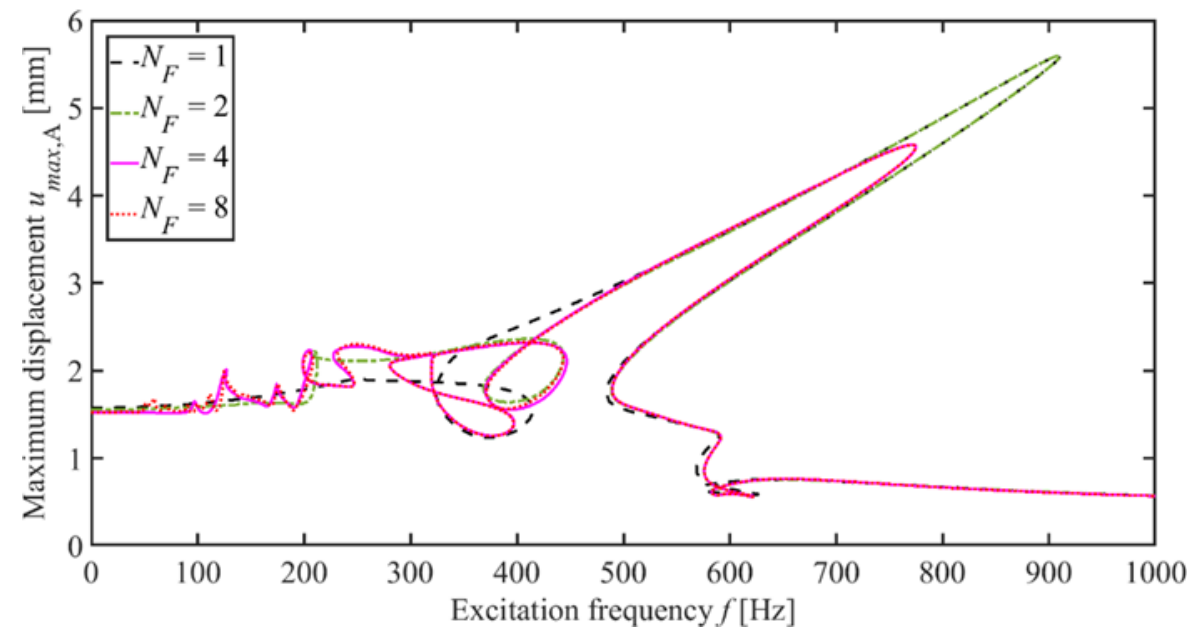

Fig. 2: Frequency response of the test problem.

\section{Conclusions}

The frequency response of a console, discretized by three-dimensional finite elements, excited by a pulsating pressure load, and mounted on a bilateral nonlinear elastic support, was investigated. The arc length continuation method was used to calculate the frequency response. The harmonic balance method was used to solve the steady-state vibration response at each increment of continuation.

\section{Acknowledgement}

This research work has been supported by the Ministry of Education, Youth and Sports from the National Programme of Sustainability (NPU II) project „IT4Innovations excellence in science - LQ1602“, by the "Student's Grant Competition project SP2020/139", and by the grant project 19-06666S of the Czech Science Foundation.

\section{References}

Batoz, J. L. and Dhatt, G. (1979) Incremental displacement algorithms for nonlinear problems. International Journal for Numerical Methods in Engineering, 14, 8, pp. 1262-1267.

Crisfield, M. A. (1981) A fast incremental/iterative solution procedure that handles "snap-through". Computers \& Structures, 13, 1-3, pp. 55-62.

Detroux, T., Renson, L., Masset, L. and Kerschen, G. (2010) The harmonic balance method for bifurcation analysis of large-scale nonlinear mechanical systems. Comp. Meth. in App. Mech. and Eng., 296, 1, pp. 18-38.

Frydrýšek, K., Michenková, Š. and Nikodým, M. (2014) Straight Beams Rested on Nonlinear Elastic Foundation Part 1 (Theory, Experiments, Numerical Approach). Applied Mechanics and Materials, 684, 1, pp. 11-20.

Krack M. and Gross, J. (2019) Harmonic Balance for Nonlinear Vibration Problems. Springer, Stuttgart. 